\title{
A New Efficient Leakage-Free Certificateless Signature
}

\author{
Zhuo Gao, a , Liang $\mathrm{Hu}^{1, \mathrm{~b}}$ and Hongtu $\mathrm{Li}^{1, \mathrm{c}}$ \\ ${ }^{1}$ College of Computer Science and Technology, Jilin University, Changchun, 130012, China \\ agaozhuo03@163.com , bhul@jlu.edu.cn, 'liht@jlu.edu.cn
}

Keywords: certificateless signature, ESL attack, computational Diffie-Hellman problem

Abstract.Digital signature, a cryptographic algorithm, provides integrity, authentication and non repudiation messages.In the literature, several certificateless signature schemes have been designed. However, most of them are vulnerable to the ephemeral secret leakage (ESL) attack.We propose a new secure certificateless signature scheme which can resist ESL attacks.Moreover, we prove the security of two types of adversaries in certificateless signature schemes. The proposed scheme provides unforgeability based on the hardness assumption of $\mathrm{CDH}$ problem.

\section{Introduction}

In 1984,Shamir first introduced the identity-based public key cryptography[1]. This algorithm uses a string generated by the user's identity information to represent his public key. Thus it can simplify the management of certificates.However, all of the user's private key are generated by the key generation center PKG, which leads to the problem of key escrow. In 2003, the certificateless public key cryptography proposed by Al-Riyami and Paterson solved this problem [2]. In this system, the user's private key is composed of two parts:a partial private key generated by KGC and the secret value selected by user.

Several certificateless signature schemes(CLS) [3-5] have been proposed,since the certificateless public key cryptography appeared. However, most of the schemes are insecure and can be attack by a public key replacement. Later, some more secure certificateless signature scheme have been proposed, which can resist the public key replacement attack to some extent [6-9]. These schemes need to generate a ephemeral secret to generate signatures. Ephemeral secrets (i.e. random values) are chosen to generate signatures, called probabilistic signatures in the sense that a signer can issue distinct signatures for the same message[11]. Note that the ephemeral secret keys $\mathrm{x}$ or $\mathrm{y}$ are not the only session-specific secret information used by the parties - they also use secret random coins in the signature generation. We observe that if the adversary reveals these random coins, it can break the security of the protocol.[15]。

If the ephemeral secret keys are compromised, an adversary can reveal the private key of the signer from the corresponding signature, termed ephemeral secret leakage (ESL) attacks[11].This attack is possible and widely studied recently in [11-14]. Since the sender must rely on internal/external source of random number generator that may be controlled by anadversary[12].Note that,the schemes above are vulnerable to the ESL attack.Note that,if the attack gets the ephemeral secret,he can compute the user's secret key and forgery use it. Therefore, the scheme above can not resist the ESL attack.In this paper we propose a new certificateless signature scheme which is able to resist ESL attack. 


\section{Preliminaries}

Bilinear Maps. Let $G_{1}$ be an additive group with prime order $q, P$ is the generator of $G_{1}$. Let $G_{2}$ be a multiplicative group with the same order.An admissible map e $: G_{1} \times G_{1} \rightarrow G_{2}$ is called a bilinear map if it satisfies the following properties[16]:

(1)Bilinearity: For any $P, Q, R \in G_{1}$, we have $e(P+Q, R)=e(P, R) e(Q, R)$ and $e(P, Q+R)=e(P, Q) e(P, R)$. In particular, for any $a, b \in Z_{q}{ }^{*}, e(a P, b P)=e(P, P){ }^{a b}=e(P, a b P)=e(a b P, P)[9]$.

(2)Non-degeneracy: There exists $P, Q \in G_{1}$, such that $e(P, Q)=1$.

(3)Computability: There is an efficient algorithm to compute $e(P, Q)$ for all $P, Q \in G_{1}$.

Security Assumption.Discrete Logarithm (DL) Problem: Given a generator $\mathrm{P}$ of a cyclic group $\mathrm{G}$ with order $\mathrm{q}$, and $\mathrm{M} \in \mathrm{G}$ to find an integer $\mathrm{a} \in \mathrm{Z}_{\mathrm{q}}{ }^{*}$, such that $\mathrm{M}=\mathrm{aP}$.

Computational Diffie-Hellman(CDH) Problem: Given a generator P of a cyclic group $\mathrm{G}$ with order $\mathrm{q}$, and given $\mathrm{aP}, \mathrm{bP}$ for unknown $\mathrm{a}, \mathrm{b} \in \mathrm{Z}_{\mathrm{q}}{ }^{*}$, to compute $\mathrm{abP}$.

Framework of Leakage-Free Certificateless Signature Schemes.A CLS consists of six algorithms [9]. The description of each algorithm is as follows.

Setup: This algorithm is run by the KGC that accepts as input a security parameter k to generate a master-key $m s k$ and a list of system parameters params.

Partial-Private-Key-Extract: This algorithm is run by the KGC that takes as input a user's identity ID, params and $m s k$ to generate the user's partial private key $D_{I D}$.

Set-Secret-Value: This algorithm is run by a user that takes as input params and a user's identity ID to generate the user's secret value $S_{I D}$.

Set-Public-Key: This algorithm is run by a user that takes as input a params, a user's identity ID and secret value $S_{I D}$ to generate the user's public key $P K_{I D}$.

Sign: This algorithm is run by a user that takes params, a message M, the user's identity ID, public key $\mathrm{PK}_{\mathrm{ID}}$, secret value $\mathrm{S}_{\mathrm{ID}}$ to produce a signature $\sigma$ on message $\mathrm{M}$.

Verify: This algorithm is run by a verifier that takes as input a message $\mathrm{M}$, a signature $\sigma$, params, a signer's identity ID and his public key $\mathrm{PK}_{\mathrm{ID}}$ and to output true if the signature is valid, or $\perp$.

Security Model for Leakage-Free Certificateless Signature Schemes.There are two types of adversaries which named Type I adversary and Type II adversary in CL-PKC.

A type I adversary $\mathrm{A}_{\mathrm{I}}$ does not have access to the master-key, but he has the ability to replace the public key of any entity with a value of his choice.

A type II adversary $A_{I I}$ has access to the master-key but cannot perform the public key replacement[16].

The security of a CLS scheme is modeled via the following two games between a challenger C and an adversary $A_{I}$ or $A_{I I}$.

\section{Leakage-Free CLS Schemes}

Our leakage-free CLS scheme consists of seven algorithms which are described as follows.

Setup: This algorithm generates the params and the master key of the system.

(1)Given a security parameter $\mathrm{k}$, the KGC chooses a cyclic additive group $\mathrm{G}_{1}$ with prime order q.P is the generator of $G_{1}$.KGC chooses a cyclic multiplicative group $G_{2}$ with the same order. and a bilinear map e : $\mathrm{G}_{1} \times \mathrm{G}_{1} \rightarrow \mathrm{G}_{2}$.

(2)The KGC also chooses a random $\mathrm{s} \in \mathrm{Z}_{\mathrm{q}}{ }^{*}$ as the master-key and sets $\mathrm{P}_{\text {pub }}=\mathrm{sP}$.

(3)KGC chooses distinct hash functions $\mathrm{H}_{1}:\{0,1\} \rightarrow \mathrm{G}_{1}, \mathrm{H}_{2}:\{0,1\} \rightarrow \mathrm{G}_{1}, \mathrm{f}_{1}:\{0,1\} \rightarrow \mathrm{Z}_{\mathrm{q}}^{*}$, 
$\mathrm{f}_{2}:\{0,1\} \rightarrow \mathrm{Z}_{\mathrm{q}}^{*}, \mathrm{f}_{3}:\{0,1\} \rightarrow \mathrm{Z}_{\mathrm{q}}^{*}$.

The system parameter list is params $=\left(\mathrm{G}_{1}, \mathrm{G}_{2}, \mathrm{e}, \mathrm{P}, \mathrm{P}_{\text {pub }}, \mathrm{H}_{1}, \mathrm{H}_{2}, \mathrm{f}_{1}, \mathrm{f}_{2}, \mathrm{f}_{3}\right)$. The master-key is $\mathrm{s}$.

Partial-Private-Key-Extract: This algorithm takes params, master-key s and a user's identity $\mathrm{ID}_{\mathrm{i}}$ $\in\{0,1\}$ as input. It generates the partial private key for the user as follows:

(1)Computes $\mathrm{Q}_{\mathrm{ID}}=\mathrm{H}_{1}\left(\mathrm{ID}_{\mathrm{i}}\right)$.

(2)Outputs the partial private key $D_{I D}=s Q_{I D}$.

It is easy to see that DID is valid on ID for the key pair $\left(\mathrm{P}_{\text {pub }}, \mathrm{s}\right)$, and user can check its correctness by checking whether $\mathrm{e}\left(\mathrm{D}_{\mathrm{ID}}, \mathrm{P}\right)=\mathrm{e}\left(\mathrm{Q}_{\mathrm{ID}}, \mathrm{P}_{\text {pub }}\right)$.

Set-Secret-Value: This algorithm takes as input params and a user's identity $\mathrm{ID}_{\mathrm{i}}$. It then selects a random $x \in Z_{q}^{*}$ and outputs $\mathrm{x}$ as the user's secret value.

Set-Public-Key: This algorithm accepts params, a user's identity $\mathrm{ID}_{\mathrm{i}}$ and this user's secret value $\mathrm{x}$ as input.It produces the user's public key $P K_{I D}=x P$.

Sign: To sign a message $\mathrm{M}$ using the partial private key $\mathrm{D}_{\mathrm{ID}}$ and the secret value $\mathrm{x}$, the signer, whose identity is $\mathrm{ID}_{\mathrm{i}}$ and the corresponding public key is $\mathrm{PK}_{\mathrm{ID}}$, performs the following steps.

(1)Choose a random $r \in Z_{q}^{*}$, compute $U=r P$.

(2)Compute $\mathrm{T}=\mathrm{f} 1$ (IDi,m,U,DID), set $R=U+T P$.

(3)Compute $V=h_{1} \times D_{I D}+(T+r) \times H I D+h_{2} \times S_{I D} \times Q_{I D}$, where $\mathrm{h}_{1}=\mathrm{f}_{2}\left(\mathrm{ID}_{\mathrm{i}}, \mathrm{m}, \mathrm{R}, \mathrm{PK} I \mathrm{ID}\right), \mathrm{h}_{2}=\mathrm{f}_{3}\left(\mathrm{ID}_{\mathrm{i}}, \mathrm{m}, \mathrm{R}\right)$ and $\mathrm{HID}=\mathrm{H}_{2}\left(\mathrm{ID}_{\mathrm{i}}, \mathrm{m}, \mathrm{R}\right)$.

(4)Output $\sigma=(R, V)$ as the signature on $M$.

Verify: To verify a signature $\sigma$ on a message $M$ for an identity $\mathrm{ID}_{\mathrm{i}}$ and public key $\mathrm{PK}_{\mathrm{ID}}$, the user performs the following steps.

(1)Compute $\mathrm{h}_{1}=\mathrm{f}_{2}\left(\mathrm{ID}_{\mathrm{i}}, \mathrm{m}, \mathrm{R}, \mathrm{PK} \mathrm{K}_{\mathrm{ID}}\right), \mathrm{h}_{2}=\mathrm{f}_{3}\left(\mathrm{ID}_{\mathrm{i}}, \mathrm{m}, \mathrm{R}\right)$ and $\mathrm{HID}=\mathrm{H}_{2}\left(\mathrm{ID}_{\mathrm{i}}, \mathrm{m}, \mathrm{R}\right)$.

(2) Verify $e(V, P)=e\left(Q I D, h_{1} \times P_{p u b}+h_{2} \times P K_{I D}\right) e(H I D, R)$.If the equation holds, output true.

Otherwise, output $\perp$.

\section{Security Proof}

Theorem 1. In the random oracle model,the proposed CLS scheme is existential unforgeable against the $\mathrm{A}_{\mathrm{I}}$ adversary assuming the $\mathrm{CDH}$ problem is intractable.

Proof. Let $\mathrm{C}$ be a $\mathrm{CDH}$ attacker who receives a random instance $(\mathrm{P}, \mathrm{aP}, \mathrm{bP})$ of the $\mathrm{CDH}$ problem in $\mathrm{G}_{1}$. Let $\mathrm{A}_{\mathrm{I}}$ be a super type $\mathrm{I}$ adversary that breaks the proposed signature. We show how $\mathrm{C}$ can use $\mathrm{A}_{\mathrm{I}}$ to solve the $\mathrm{CDH}$ problem, that is to compute abP.

First $\mathrm{C}$ sets $\mathrm{P}_{\mathrm{pub}}=\mathrm{aP}$, where $\mathrm{P}_{\mathrm{pub}}$ is the public key of the KGC.Then $\mathrm{C}$ selects params $=\left\{\mathrm{G}_{1}, \mathrm{G}_{2}, \mathrm{e}, \mathrm{q}, \mathrm{P}, \mathrm{P}_{\mathrm{pub}}, \mathrm{H}_{1}, \mathrm{H}_{2}, \mathrm{f}_{1}, \mathrm{f}_{2}, \mathrm{f}_{3}\right\}$ and sends it to $\mathrm{A}_{\mathrm{I}}$.

$\mathrm{H}_{1}$ Queries:Suppose $\mathrm{A}_{\mathrm{I}}$ can make at most $\mathrm{qH}_{1}$ times $\mathrm{H}_{1}$ queries, $\mathrm{C}$ chooses $j \in\left[1, \mathrm{qH}_{1}\right] . \mathrm{C}$ maintains an initially empty list $\mathrm{H}_{1}^{\text {list }}$ of tuples $\left(\mathrm{ID}_{\mathrm{j}}, \mathrm{QID}_{\mathrm{j}}, \alpha_{\mathrm{j}}\right)$. On receiving a new query $\mathrm{H}_{1}\left(\mathrm{ID}_{\mathrm{i}}\right), \mathrm{C}$ responds as follows:

(1)If $\mathrm{ID}_{\mathrm{i}}$ has appeared on the $\mathrm{H}_{1}{ }^{\text {list }}$, then $\mathrm{C}$ responds with $\mathrm{H}_{1}\left(\mathrm{ID}_{\mathrm{i}}\right)=\mathrm{Q}_{\mathrm{ID}} \in \mathrm{G}_{1}$.

(2)If $\mathrm{ID}_{\mathrm{i}}$ has not appeared on the list, then $\mathrm{C}$ selects $\alpha \in \mathrm{Z}_{\mathrm{q}}{ }^{*}$ at random.

a.If $\mathrm{i}=j$,Set $\mathrm{H}_{1}\left(\mathrm{ID}_{\mathrm{i}}\right)=\mathrm{Q}_{\mathrm{ID}}=\alpha \mathrm{bP}$.Return $\mathrm{Q}_{\mathrm{ID}}$ as answer.

b. Otherwise, set $\mathrm{H}_{1}\left(\mathrm{ID}_{\mathrm{i}}\right)=\mathrm{Q}_{\text {ID }}=\alpha \mathrm{P}$.

Adds $\left(\mathrm{ID}_{\mathrm{i}}, \mathrm{Q}_{\mathrm{ID}}, \alpha\right)$ to $\mathrm{H}_{1}{ }^{\text {list }}$ and return $\mathrm{Q}_{\mathrm{ID}}$ as respond.

Partial-Private-Key Queries:C keeps an initially empty list $\mathrm{K}^{\text {list }}$ of tuples $\left(\mathrm{ID}_{\mathrm{j}}, \mathrm{PK}_{\mathrm{IDj}}, \mathrm{S}_{\mathrm{IDj}}, \mathrm{D}_{\mathrm{IDj}}\right)$. When $\mathrm{A}_{\mathrm{I}}$ issues a query on $\mathrm{ID}_{\mathrm{i}}, \mathrm{C}$ responds as follows: 
(1)If $\mathrm{ID}_{\mathrm{i}}$ has appeared on the $\mathrm{K}^{\text {list }}$, then $\mathrm{C}$ responds with $\mathrm{D}_{\mathrm{ID}}$.

(2)If $\mathrm{ID}_{\mathrm{i}}$ has not appeared on the list,then $\mathrm{C}$ finds for $\mathrm{H}_{1}{ }^{\text {list }}$ the tuple $\left(\operatorname{ID}_{\mathrm{i}}, \mathrm{QID}_{\mathrm{i}}, \mathrm{\alpha}_{\mathrm{i}}\right)$.

a.If there is a tuple $\left(\operatorname{ID}_{\mathrm{i}}, \mathrm{QID}, \alpha_{\mathrm{i}}\right)$ on the H1list and $\operatorname{ID}_{\mathrm{i}}=\mathrm{ID}_{\mathrm{j}}$, abort.

b.If $\mathrm{ID}_{\mathrm{i}} \neq \mathrm{ID}_{\mathrm{j}}$, set $\mathrm{D}_{\mathrm{ID}}=\alpha_{\mathrm{i}} \mathrm{P}_{\mathrm{pub}}=\alpha_{\mathrm{i}} \times \mathrm{aP}, \mathrm{S}_{\mathrm{ID}}=\perp$ and $\mathrm{PK}_{\mathrm{ID}}=\perp$.

c. Otherwise, set $\mathrm{D}_{\mathrm{ID}}=\alpha_{\mathrm{i}} \mathrm{P}_{\mathrm{pub}}=\alpha_{\mathrm{i}} \times \mathrm{aP}, \mathrm{S}_{\mathrm{ID}}=\perp$ and $\mathrm{PK}_{\mathrm{ID}}=\perp$.

Adds $\left(\mathrm{ID}_{\mathrm{i}}, \mathrm{PK}_{\mathrm{ID}}, \mathrm{S}_{\mathrm{ID}}, \mathrm{D}_{\mathrm{ID}}\right)$ to $\mathrm{K}^{\text {list }}$ and return $\mathrm{D}_{\mathrm{ID}}$ as respond.

$\mathrm{H}_{2}$ Queries: $\mathrm{C}$ keeps an initially empty list $\mathrm{H}_{2}{ }^{\text {list }}$ of tuples $\left(\mathrm{ID}_{\mathrm{j}}, \mathrm{m}_{\mathrm{j}}, \mathrm{U}_{\mathrm{j}}, \mathrm{h}_{2}\right)$. Whenever $\mathrm{A}_{\mathrm{I}}$ issues a query $\left(\mathrm{ID}_{\mathrm{i}}, \mathrm{m}_{\mathrm{i}}, \mathrm{U}_{\mathrm{i}}\right)$ to $\mathrm{H}_{2}$, the same answer from the list $\mathrm{H}_{2}$ will be given if the request has been asked before. If the query $\left(\mathrm{ID}_{\mathrm{i}}, \mathrm{m}_{\mathrm{i}}, \mathrm{U}_{\mathrm{i}}\right)$ is new, $\mathrm{C}$ selects a random $\beta \in \mathrm{Z}_{\mathrm{q}}{ }^{*}$ and set $\mathrm{h}_{2}=\beta \mathrm{P}$.Adds $\left(\mathrm{ID}_{\mathrm{i}}, \mathrm{m}_{\mathrm{i}}, \mathrm{U}_{\mathrm{i}}, \mathrm{h}_{2}\right)$ to $\mathrm{H}_{2}{ }^{\text {list }}$ and returns $\mathrm{h}_{2}$ as answer.

$\mathrm{f}_{1}$ Queries: C keeps an initially empty list $\mathrm{f}_{1}$ list of tuples $\left(\mathrm{ID}_{\mathrm{j}}, \mathrm{m}_{\mathrm{j}}, \mathrm{U}_{\mathrm{j}}, \mathrm{D}_{\mathrm{IDj}}, \mathrm{f}_{1}\right)$. Whenever $\mathrm{A}_{\mathrm{I}}$ issues a query $\left(\mathrm{ID}_{\mathrm{i}}, \mathrm{m}_{\mathrm{i}}, \mathrm{U}_{\mathrm{i}}, \mathrm{D}_{\mathrm{IDi}}\right)$ to $\mathrm{f}_{1}$, the same answer from the list $\mathrm{f}_{1}$ list will be given if the request has been asked before. If the query $\left(\mathrm{ID}_{\mathrm{i}}, \mathrm{m}_{\mathrm{i}}, \mathrm{U}_{\mathrm{i}}, \mathrm{D}_{\mathrm{IDi}}\right)$ is new, $\mathrm{C}$ selects a random $\mathrm{t} \in \mathrm{Z}_{\mathrm{q}}{ }_{\mathrm{q}}$ and set $\mathrm{fl}=\mathrm{t}$.Adds $\left(\mathrm{ID}_{\mathrm{i}}, \mathrm{m}_{\mathrm{i}}, \mathrm{U}_{\mathrm{i}}, \mathrm{D}_{\mathrm{IDi}}, \mathrm{f}_{1}\right)$ to $\mathrm{f}_{1}{ }^{\text {list }}$ and returns $\mathrm{f}_{1}$ as answer.

$\mathrm{f}_{2}$ Queries: $\mathrm{C}$ keeps an initially empty list $\mathrm{f}_{2}^{\text {list }}$ of tuples $\left(\mathrm{ID}_{\mathrm{j}}, \mathrm{m}_{\mathrm{j}}, \mathrm{R}_{\mathrm{j}}, \mathrm{PK}_{\mathrm{IDj}}, \mathrm{f}_{2}\right)$. Whenever $\mathrm{A}_{\mathrm{I}}$ issues a query $\left(\mathrm{ID}_{\mathrm{i}}, \mathrm{m}_{\mathrm{i}}, \mathrm{R}_{\mathrm{i}}, \mathrm{PK} \mathrm{K}_{\mathrm{IDi}}\right)$ to $\mathrm{f}_{2}$, the same answer from the list $\mathrm{f}_{2}{ }^{\text {list }}$ will be given if the request has been asked before. If the query $\left(\mathrm{ID}_{\mathrm{i}}, \mathrm{m}_{\mathrm{i}}, \mathrm{R}_{\mathrm{i}}, \mathrm{PK}\right.$ IDi $)$ is new, $\mathrm{C}$ selects a random $\mathrm{u} \in \mathrm{Z}_{\mathrm{q}}{ }^{*}$ and set $\mathrm{f}_{2}=\mathrm{u}$.Adds $\left(\left(\mathrm{ID}_{\mathrm{i}}, \mathrm{m}_{\mathrm{i}}, \mathrm{R}_{\mathrm{i}}, \mathrm{PK}_{\mathrm{IDi}}, \mathrm{f}_{2}\right)\right.$ to $\mathrm{f}_{2}$ list and returns $\mathrm{f}_{2}$ as answer.

$\mathrm{f}_{3}$ Queries: $C$ keeps an initially empty list $\mathrm{f}_{3}$ list of tuples $\left(\mathrm{ID}_{\mathrm{j}}, \mathrm{m}_{\mathrm{j}}, \mathrm{R}_{\mathrm{j}}, \mathrm{f}_{3}\right)$. Whenever $\mathrm{A}_{\mathrm{I}}$ issues a query $\left(\mathrm{ID}_{\mathrm{i}}, \mathrm{m}_{\mathrm{i}}, \mathrm{R}_{\mathrm{i}}\right)$ to $\mathrm{f}_{3}$, the same answer from the list $\mathrm{f}_{3}$ list will be given if the request has been asked before. If the query $\left(I_{i}, m_{i}, R_{i}\right)$ is new, $C$ selects a random $v \in Z_{q}^{*}$ and set $f_{3}=v$.Adds $\left(\operatorname{ID}_{i}, m_{i}, R_{i}, f_{3}\right)$ to $\mathrm{f}_{3}{ }^{\text {list }}$ and returns $\mathrm{f}_{3}$ as answer.

Public-Key Queries: When $\mathrm{A}_{\mathrm{I}}$ issues a query on $\mathrm{ID}_{\mathrm{i}}, \mathrm{C}$ responds as follows:

(1)If $\mathrm{ID}_{\mathrm{i}}$ has appeared on the $\mathrm{K}^{\text {list }}$ and $\mathrm{PK}_{\mathrm{IDi}} \neq \perp$, then $\mathrm{C}$ responds with $\mathrm{PK} \mathrm{IDi}$.

(2)If $\mathrm{PK}_{\mathrm{ID}}=\perp$, select $\mathrm{x}, \in \mathrm{Z}_{\mathrm{q}}{ }^{*}$ in random.Set $\mathrm{S}_{\mathrm{ID}}=\mathrm{x}$ ' and $\mathrm{PK} \mathrm{K}_{\mathrm{ID}}=\mathrm{x}$ 'P.Then $\mathrm{C}$ responds with $\mathrm{PK}_{\mathrm{ID}}$.

(3)Otherwise, select $\mathrm{x} \in \mathrm{Z}_{\mathrm{q}}{ }^{*}$ in random. Set $\mathrm{D}_{\mathrm{ID}}=\perp, \mathrm{S}_{\mathrm{ID}}=\mathrm{x}$ and $\mathrm{PK} \mathrm{K}_{\mathrm{ID}}=\mathrm{xP}$.Adds $\left(\mathrm{ID}_{\mathrm{i}}, \mathrm{PK}_{\mathrm{ID}}, \mathrm{S}_{\mathrm{ID}}, \mathrm{D}_{\mathrm{ID}}\right)$ to $\mathrm{K}^{\text {list }}$ and return $\mathrm{PK}_{\mathrm{ID}}$ as respond.

Public-Key-Replacement Queries: $\mathrm{A}_{\mathrm{I}}$ can choose a new public key $\mathrm{PK}_{\mathrm{ID}}$ ' for the user whose identity is $\mathrm{ID}_{\mathrm{i}} . \mathrm{C}$ first finds the list $\mathrm{K}^{\text {list }}$. If $\mathrm{ID}_{\mathrm{i}}$ has appeared on the list and $\mathrm{PK}_{\mathrm{ID}} \neq \perp$, then set $\mathrm{D}_{\mathrm{ID}}=\perp, \mathrm{S}_{\mathrm{ID}}=\perp$ and $\mathrm{PK}_{\mathrm{ID}}=\mathrm{PK}_{\mathrm{ID}}$ '. Otherwise, make Public-Key Queries, then update $\mathrm{PK}_{\mathrm{ID}}=\mathrm{PK}_{\mathrm{ID}}$ '. Adds $\left(\mathrm{ID}_{\mathrm{i}}, \mathrm{PK}_{\mathrm{ID}}, \mathrm{S}_{\mathrm{ID}}, \mathrm{D}_{\mathrm{ID}}\right)$ to $\mathrm{K}^{\text {list }}$.

Secret-Value Queries: Suppose the query is on $\mathrm{ID}_{\mathrm{i}} \cdot \mathrm{C}$ find the list $\mathrm{K}^{\text {list }}$.If IDi has appeared on the list and $\mathrm{S}_{\mathrm{ID}}=\perp$, it means that the public key of $\mathrm{ID}_{\mathrm{i}}$ has been replaced.C returns $\perp$.Else, $\mathrm{C}$ returns $\mathrm{S}_{\mathrm{ID}}$.If $\mathrm{ID}_{\mathrm{i}}$ has not appeared on the list,C makes Public-Key Queries on $\mathrm{ID}_{\mathrm{i}}$ and returns the corresponding $\mathrm{S}_{\mathrm{ID}}$ as answer.

Sign Queries: Note that at any time during the simulation, equipped with those partial private keys for any $I D_{i} \neq I D_{j}, A_{I}$ is able to generate signatures on any message. If $\operatorname{ID}_{i}=I_{j}, A_{I}$ issues a query $\left(\mathrm{mi}, \mathrm{PK}_{\mathrm{IDi}}\right)$ where $\mathrm{m}_{\mathrm{i}}$ means a message and $\mathrm{PK}_{\mathrm{IDi}}$ means a current public key chosen by $\mathrm{A}_{\mathrm{I}}$ to the signature whose private key is associated with $\mathrm{ID}_{\mathrm{j}}$. On receiving this, $\mathrm{C}$ creates a signature as follows:

(1)Select $\mathrm{k}, \mathrm{u}, \mathrm{v} \in \mathrm{Z}_{\mathrm{q}}{ }^{*}$ in random.Set $\mathrm{U}_{\mathrm{i}}=\mathrm{P}-\mathrm{kP}$ and $\mathrm{R}_{\mathrm{i}}=\mathrm{kP}$.

(2) Compute $\mathrm{V}_{\mathrm{i}}=\alpha_{\mathrm{i}} \mathrm{b}\left(\mathrm{uP} \mathrm{P}_{\text {pub }}+\mathrm{vPK} \mathrm{K}_{\mathrm{IDi}}\right)+\beta \mathrm{P}$. Output the signature $\sigma_{\mathrm{i}}=\left(\mathrm{R}_{\mathrm{i}}, \mathrm{V}_{\mathrm{i}}\right)$.

Forgery: Finally, $\mathrm{A}_{\mathrm{I}}$ outputs a signature $\left(\mathrm{M}, \sigma=(\mathrm{R}, \mathrm{V}), \mathrm{ID}, \mathrm{PK}_{\mathrm{ID}}{ }^{*}\right)$ which means $(\mathrm{R}, \mathrm{V})$ is a valid signature on message $M$ for identity ID and public key $\mathrm{PK}_{\mathrm{ID}}{ }^{*}$. If $\mathrm{ID}^{*} \neq \mathrm{ID}_{\mathrm{j}}, \mathrm{C}$ aborts. By forking lemma [17], $\mathrm{C}$ replays $\mathrm{A}_{\mathrm{I}}$ with the same random tape but different choice of the hash 
function $\mathrm{h}_{1}$ for another choice of $\mathrm{f}_{2}$.Now $\mathrm{A}_{\mathrm{I}}$ outputs another forged signature $\left(\mathrm{M}, \sigma=\left(\mathrm{R}, \mathrm{V}^{\prime}\right), \mathrm{ID}, \mathrm{PK}_{\mathrm{ID}}{ }^{*}\right)$. Both of the two signatures are valid,so they should satisfy the following equations.

$$
\begin{aligned}
& \mathrm{e}\left(\mathrm{V}^{*}, \mathrm{P}\right)=\mathrm{e}\left(\mathrm{Q}_{\mathrm{ID}}{ }^{*}, \mathrm{~h}_{1}{ }^{*} \times \mathrm{P}_{\mathrm{pub}}+\mathrm{h}_{2}{ }^{*} \times \mathrm{PK}_{\mathrm{ID}}{ }^{*}\right) \mathrm{e}\left(\mathrm{HID}^{*}, \mathrm{R}^{*}\right) \\
& \mathrm{e}(\mathrm{V} ; \mathrm{P})=\mathrm{e}\left(\mathrm{Q}_{\mathrm{ID}}{ }^{*}{ } \mathrm{~h}_{1}{ } \times \mathrm{P}_{\mathrm{pub}}+\mathrm{h}_{2}{ }^{*} \times \mathrm{PK}_{\mathrm{ID}}{ }^{*}\right) \mathrm{e}\left(\mathrm{HID}^{*}, \mathrm{R}^{*}\right)
\end{aligned}
$$

Where $\mathrm{P}_{\text {pub }}=\mathrm{aP}, \mathrm{Q}_{\mathrm{ID}}{ }^{*}=\alpha \times \mathrm{bP}, \mathrm{HID}=\beta^{*} \mathrm{P}$ and $\mathrm{h}_{1}{ }^{*} \neq \mathrm{h}_{1}{ }^{\prime}$. Hence we have $\mathrm{V}^{*}=\alpha \times \mathrm{b} \times \mathrm{h}_{1}{ }^{*} \times \mathrm{aP}+\alpha \times \mathrm{b} \times \mathrm{h}_{2}{ }^{*}$ $\times \mathrm{PK}_{\mathrm{ID}}{ }^{*}+\beta^{*} \mathrm{R}^{*}$ and $\mathrm{V}^{\prime}=\alpha \times \mathrm{b} \times \mathrm{h}_{1}{ }^{*} \times \mathrm{aP}+\alpha \times \mathrm{h}_{2}{ }^{*} \times \mathrm{PK}_{\mathrm{ID}}{ }^{*}+\beta \mathrm{R}^{*}$. C can compute $\mathrm{abP}=\left(\mathrm{V}^{*}-\mathrm{V}^{\prime}\right) /\left(\alpha \times\left(\mathrm{h}_{1}{ }^{*}-\mathrm{h}_{1}{ }^{\prime}\right)\right)$.So $\mathrm{C}$ can successfully obtain the solution of the $\mathrm{CDH}$ problem.

Theorem 2. In the random oracle model,the proposed CLS scheme is existential unforgeable against the $\mathrm{A}_{\mathrm{II}}$ adversary assuming the $\mathrm{CDH}$ problem is intractable.

Proof. Let $\mathrm{C}$ be a $\mathrm{CDH}$ attacker who receives a random instance $(\mathrm{P}, \mathrm{aP}, \mathrm{bP})$ of the $\mathrm{CDH}$ problem in $\mathrm{G}_{1}$.Let $\mathrm{A}_{\mathrm{II}}$ be a super type II adversary that breaks the proposed signature. We show how $\mathrm{C}$ can use $\mathrm{A}_{\text {II }}$ to solve the $\mathrm{CDH}$ problem, that is to compute abP.

First $\mathrm{C}$ selects a random $\mathrm{s} \in \mathrm{Z}_{\mathrm{q}}{ }^{*}$ as the master-key and sets $\mathrm{P}_{\mathrm{pub}}=\mathrm{sP}$, where Ppub is the public key of the KGC.Then $C$ selects params $=\left\{G_{1}, G_{2}, e, q, P, P_{\text {pub }}, H_{1}, H_{2}, f_{1}, f_{2}, f_{3}\right\}$. And initializes $A_{I I}$ with the params and the masker-key s.

$\mathrm{H}_{1}$ Queries:Suppose $A_{\text {II }}$ can make at most $\mathrm{qH}_{1}$ times $\mathrm{H}_{1}$ queries, $\mathrm{C}$ chooses $\mathrm{j} \in\left[1, \mathrm{qH}_{1}\right]$.C maintains an initially empty list $\mathrm{H}_{1}{ }^{\text {list }}$ of tuples $\left(\mathrm{ID}_{\mathrm{j}}, \mathrm{Q}_{\mathrm{IDj}}, \mathrm{\alpha}_{\mathrm{j}}\right)$. On receiving a new query $\mathrm{H}_{1}\left(\mathrm{ID}_{\mathrm{i}}\right), \mathrm{C}$ responds as follows:

(1)If $\mathrm{ID}_{\mathrm{i}}$ has appeared on the $\mathrm{H}_{1}{ }^{\text {list }}$, then $\mathrm{C}$ responds with $\mathrm{H} 1(\mathrm{IDi})=\mathrm{Q}_{\mathrm{ID}} \in \mathrm{G}_{1}$.

(2)If $\mathrm{ID}_{\mathrm{i}}$ has not appeared on the list, then $\mathrm{C}$ selects $\alpha \in \mathrm{Z}_{\mathrm{q}}{ }^{*}$ at random.Set $\mathrm{Q}_{\mathrm{ID}}=\mathrm{H}_{1}\left(\mathrm{ID}_{\mathrm{i}}\right)=\alpha \mathrm{aP}$.Adds (ID, $\left.\mathrm{Q}_{\mathrm{ID}}, \alpha\right)$ to $\mathrm{H}_{1}{ }^{\text {list }}$ and return $\mathrm{Q}_{\mathrm{ID}}$ as respond.

Partial-Private-Key Queries:C keeps an initially empty list $\mathrm{K}^{\text {list }}$ of tuples $\left(\mathrm{ID}_{\mathrm{j}}, \mathrm{PK}_{\mathrm{IDj}}, \mathrm{S}_{\mathrm{IDj}}\right)$. When $\mathrm{A}_{\mathrm{II}}$ issues a query on $\mathrm{ID}_{\mathrm{i}}, \mathrm{C}$ responds as follows:

(1)If $\mathrm{ID}_{\mathrm{i}}$ has appeared on the $\mathrm{K}^{\text {list }}$, then $\mathrm{C}$ responds with $\mathrm{D}_{\mathrm{ID}}$.

(2)If $\mathrm{ID}_{\mathrm{i}}$ has not appeared on the list, then $\mathrm{C}$ finds for $\mathrm{H}_{1}{ }^{\text {list }}$ the tuple $\left(\mathrm{ID}_{\mathrm{i}}, \mathrm{Q}_{\mathrm{ID}}, \alpha_{\mathrm{i}}\right)$.

a.If there is a tuple $\left(\mathrm{ID}_{\mathrm{i}}, \mathrm{Q}_{\mathrm{ID}}, \alpha_{\mathrm{i}}\right)$ on the $\mathrm{H}_{1}{ }^{\text {list }}$, then set $\mathrm{D}_{\mathrm{ID}}=\mathrm{s} \mathrm{Q}_{\mathrm{ID}}=\alpha_{\mathrm{i}} \times \mathrm{aP}_{\text {pub }}$.

b. Otherwise, set $\mathrm{DID}=\mathrm{s} \mathrm{Q}_{\mathrm{ID}}=\alpha_{\mathrm{i}} \times \mathrm{aP}_{\mathrm{pub}}, \mathrm{S}_{\mathrm{ID}}=\perp$ and $\mathrm{PK} \mathrm{ID}=\perp$.

Adds $\left(\mathrm{ID}_{\mathrm{i}}, \mathrm{PK}_{\mathrm{ID}}, \mathrm{S}_{\mathrm{ID}}, \mathrm{D}_{\mathrm{ID}}\right)$ to $\mathrm{K}^{\text {list }}$ and return $\mathrm{D}_{\mathrm{ID}}$ as respond.

$\mathrm{H}_{2}$ Queries: $\mathrm{C}$ keeps an initially empty list $\mathrm{H}_{2}{ }^{\text {list }}$ of tuples $\left(\mathrm{ID}_{\mathrm{j}}, \mathrm{m}_{\mathrm{j}}, \mathrm{U}_{\mathrm{j}}, \mathrm{h}_{2}\right)$. Whenever $\mathrm{A}_{\mathrm{II}}$ issues a query $\left(\mathrm{ID}_{\mathrm{i}}, \mathrm{m}_{\mathrm{i}}, \mathrm{U}_{\mathrm{i}}\right)$ to $\mathrm{H}_{2}$, the same answer from the list $\mathrm{H}_{2}$ will be given if the request has been asked before. If the query $\left(\operatorname{ID}_{\mathrm{i}}, \mathrm{m}_{\mathrm{i}}, \mathrm{U}_{\mathrm{i}}\right)$ is new, $\mathrm{C}$ selects a random $\beta \in \mathrm{Z}_{\mathrm{q}}{ }^{*}$ and set $\mathrm{h}_{2}=\beta \mathrm{P}$.Adds $\left(\left(\mathrm{ID}_{\mathrm{i}}, \mathrm{m}_{\mathrm{i}}, \mathrm{U}_{\mathrm{i}}, \mathrm{h}_{2}\right)\right.$ to $\mathrm{H}_{2}{ }^{\text {list }}$ and returns $\mathrm{h}_{2}$ as answer.

$\mathrm{f}_{1}$ Queries: $\mathrm{C}$ keeps an initially empty list $\mathrm{f}_{1}{ }^{\text {list }}$ of tuples $\left(\mathrm{ID}_{\mathrm{j}}, \mathrm{m}_{\mathrm{j}}, \mathrm{U}_{\mathrm{j}}, \mathrm{DID}_{\mathrm{j}}, \mathrm{f}_{1}\right)$. Whenever $\mathrm{A}_{\mathrm{II}}$ issues a query $\left(\mathrm{ID}_{\mathrm{i}}, \mathrm{m}_{\mathrm{i}}, \mathrm{U}_{\mathrm{i}}, \mathrm{DID}_{\mathrm{i}}\right)$ to $\mathrm{f}_{1}$, the same answer from the list $\mathrm{f}_{1}$ list will be given if the request has been asked before. If the query $\left(\mathrm{ID}_{\mathrm{i}}, \mathrm{m}_{\mathrm{i}}, \mathrm{U}_{\mathrm{i}}, \mathrm{DID}_{\mathrm{i}}\right)$ is new, $\mathrm{C}$ selects a random $\mathrm{t} \in \mathrm{Z}_{\mathrm{q}}{ }_{\mathrm{q}}$ and set $\mathrm{f}_{1}=\mathrm{t}$.Adds $\left(\left(\mathrm{ID}_{\mathrm{i}}, \mathrm{m}_{\mathrm{i}}, \mathrm{U}_{\mathrm{i}}, \mathrm{DID}_{\mathrm{i}}, \mathrm{f}_{1}\right)\right.$ to $\mathrm{f}_{1}{ }^{\text {list }}$ and returns $\mathrm{f}_{1}$ as answer.

$\mathrm{f}_{2}$ Queries: $\mathrm{C}$ keeps an initially empty list $\mathrm{f}_{2}{ }^{\text {list }}$ of tuples $\left(\mathrm{ID}_{\mathrm{j}}, \mathrm{m}_{\mathrm{j}}, \mathrm{R}_{\mathrm{j}}, \mathrm{PK}_{\mathrm{IDj}}, \mathrm{f}_{2}\right)$. Whenever $\mathrm{A}_{\mathrm{II}}$ issues a query $\left(\mathrm{ID}_{\mathrm{i}}, \mathrm{m}_{\mathrm{i}}, \mathrm{R}_{\mathrm{i}}, \mathrm{PK}_{\mathrm{IDi}}\right)$ to $\mathrm{f}_{2}$, the same answer from the list $\mathrm{f}_{2}$ list will be given if the request has been asked before. If the query $\left(\mathrm{ID}_{\mathrm{i}}, \mathrm{m}_{\mathrm{i}}, \mathrm{R}_{\mathrm{i}}, \mathrm{PK} \mathrm{K}_{\mathrm{IDi}}\right)$ is new, $\mathrm{C}$ selects a random $\mathrm{u} \in \mathrm{Z}_{\mathrm{q}}{ }^{*}$ and set $f_{2}=$ u.Adds $\left(I D_{j}, m_{j}, R_{j}, P K_{I D j}, f_{2}\right)$ to $f_{2}{ }^{\text {list }}$ and returns $f_{2}$ as answer.

$\mathrm{f}_{3}$ Queries: $\mathrm{C}$ keeps an initially empty list $\mathrm{f}_{3}{ }^{\text {list }}$ of tuples $\left(\mathrm{ID}_{\mathrm{j}}, \mathrm{m}_{\mathrm{j}}, \mathrm{R}_{\mathrm{j}}, \mathrm{f}_{3}\right)$. Whenever $\mathrm{A}_{\mathrm{II}}$ issues a 
query $\left(\mathrm{ID}_{\mathrm{i}}, \mathrm{m}_{\mathrm{i}}, \mathrm{R}_{\mathrm{i}}\right)$ to $\mathrm{f}_{3}$, the same answer from the list $\mathrm{f}_{3}{ }^{\text {list }}$ will be given if the request has been asked before. If the query $\left(I_{i}, m_{i}, R_{i}\right)$ is new, $C$ selects a random $v \in Z_{q}{ }^{*}$ and set $f_{3}=v$.Adds $\left(I_{i}, m_{i}, R_{i}, f_{3}\right)$ to $\mathrm{f}_{3}{ }^{\text {list }}$ and returns $\mathrm{f}_{3}$ as answer.

Public-Key Queries: When $\mathrm{A}_{\mathrm{II}}$ issues a query on $\mathrm{ID}_{\mathrm{i}}, \mathrm{C}$ responds as follows:

(1)If $\mathrm{ID}_{\mathrm{i}}$ has appeared on the $K^{\text {list }}$ and $\mathrm{PK}_{\mathrm{ID}} \neq \perp$, then $\mathrm{C}$ responds with $\mathrm{PK}_{\mathrm{ID}}$.

(2)If $\mathrm{ID}_{\mathrm{i}}$ has not appeared on the list and $\mathrm{ID}_{\mathrm{i}}=\mathrm{ID}_{\mathrm{j}}$, select ${ }_{\mathrm{W}} \in \mathrm{Z}_{\mathrm{q}}{ }^{*}$ in random.Set $\mathrm{S}_{\mathrm{ID}}=\mathrm{W}$ and $\mathrm{PK}_{\mathrm{ID}}=\mathrm{w} \times \mathrm{bP}$.Adds (ID, $\left.\mathrm{PK}_{\mathrm{ID}}, \mathrm{S}_{\mathrm{ID}}\right)$ to $\mathrm{K}^{\text {list }}$ and return $\mathrm{PK}_{\mathrm{ID}}$ as respond.

(3)Otherwise, select $\mathrm{w} \in \mathrm{Z}_{\mathrm{q}}{ }^{*}$ in random.Set $\mathrm{S}_{\mathrm{ID}}=\mathrm{w}$ and $\mathrm{PK} \mathrm{ID}=\mathrm{wP}$.Adds (ID,PK $\mathrm{ID}_{\mathrm{ID}}, \mathrm{S}_{\mathrm{ID}}$ ) to $\mathrm{K}^{\text {list }}$ and return $\mathrm{PK}_{\mathrm{ID}}$ as respond.

Secret-Value Queries: Suppose the query is on IDi.C find the list $K^{\text {list }}$.If IDi has appeared on the list and $\mathrm{ID}_{\mathrm{i}} \neq \mathrm{ID}_{\mathrm{j}}, \mathrm{C}$ returns $\perp$.Otherwise, $\mathrm{C}$ return $\mathrm{S}_{\mathrm{ID}}$.If IDi has not appeared on the list, $\mathrm{C}$ makes Public-Key Queries on $\mathrm{ID}_{\mathrm{i}}$ and returns the corresponding $\mathrm{S}_{\mathrm{ID}}$ as answer.

Sign Queries: Note that at any time during the simulation, equipped with those partial private keys for any $\mathrm{ID}_{\mathrm{i}} \neq \mathrm{ID}_{\mathrm{j}}, \mathrm{A}_{\mathrm{II}}$ is able to generate signatures on any message. If $\mathrm{ID}_{\mathrm{i}}=\mathrm{ID}_{\mathrm{j}}, \mathrm{A}_{\mathrm{II}}$ issues a query $\left(\mathrm{m}_{\mathrm{i}}, \mathrm{PK}_{\mathrm{IDi}}\right)$ where mi means a message and $\mathrm{PK}_{\mathrm{IDi}}$ means a current public key chosen by $\mathrm{A}_{\mathrm{II}}$ to the signature whose private key is associated with $\mathrm{ID}_{\mathrm{j}}$. When $\mathrm{C}$ receives this, $\mathrm{C}$ creates a signature as follows:

(1)Select $\mathrm{k}, \mathrm{u}, \mathrm{v} \in \mathrm{Z}_{\mathrm{q}}^{*}$ in random.Set $\mathrm{Ui}=\mathrm{P}-\mathrm{kP}$ and $\mathrm{Ri}=\mathrm{kP}$.

(2)Compute $\mathrm{V}_{\mathrm{i}}=\alpha_{\mathrm{i}} \mathrm{b}\left(\mathrm{uP} \mathrm{P}_{\text {pub }}+\mathrm{vPK} \mathrm{K}_{\mathrm{IDi}}\right)+\beta \mathrm{P}$. Output the signature $\sigma_{\mathrm{i}}=\left(\mathrm{R}_{\mathrm{i}}, \mathrm{V}_{\mathrm{i}}\right)$.

Forgery: Finally, $A_{\text {II }}$ outputs a signature $\left(M, \sigma=(R, V), I D, \mathrm{PK}_{I D}{ }^{*}\right)$ which means $(R, V)$ is a valid signature on message $M$ for identity ID and public key PKID*. If ID $^{*} \neq \mathrm{ID}_{\mathrm{j}}, \mathrm{C}$ aborts. By forking lemma [17], $\mathrm{C}$ replays $\mathrm{A}_{\mathrm{II}}$ with the same random tape but different choice of the hash function $h_{2}$ for another choice of $f_{3}$. Now $A_{\text {II }}$ outputs another forged signature $\left(M, \sigma \sigma^{\prime}=\left(R, V^{\prime}\right), I D\right.$, $\left.\mathrm{PK}_{\mathrm{ID}}{ }^{*}\right)$. Both of the two signatures are valid,so they should satisfy the following equations.

$$
\begin{aligned}
& \mathrm{e}\left(\mathrm{V}^{*}, \mathrm{P}\right)=\mathrm{e}\left(\mathrm{Q}_{\mathrm{ID}}{ }^{*}, \mathrm{~h}_{1}{ }^{*} \times \mathrm{P}_{\mathrm{pub}}+\mathrm{h}_{2}{ }^{*} \times \mathrm{PK}_{\mathrm{ID}}{ }^{*}\right) \mathrm{e}\left(\mathrm{HID}^{*}, \mathrm{R}^{*}\right) \\
& \mathrm{e}\left(\mathrm{V}^{\prime}, \mathrm{P}\right)=\mathrm{e}\left(\mathrm{Q}_{\mathrm{ID}}{ }^{*}, \mathrm{~h}_{1}{ }^{*} \times \mathrm{P}_{\mathrm{pub}}+\mathrm{h}_{2}{ } \times \mathrm{PK}_{\mathrm{ID}}{ }^{*}\right) \mathrm{e}\left(\mathrm{HID}^{*}, \mathrm{R}^{*}\right)
\end{aligned}
$$

Where $\mathrm{Q}_{\mathrm{ID}}{ }^{*}=\alpha \times \mathrm{aP}, \mathrm{PK}_{\mathrm{ID}}{ }^{*}=\mathrm{w} \times \mathrm{bP}, \mathrm{HID}=\beta^{*} \mathrm{P}$ and $\mathrm{h}_{2}{ }^{*} \neq \mathrm{h}_{2}$. Hence we have $\mathrm{V}^{*}=\alpha \times \mathrm{a} \times \mathrm{h}_{1}{ }^{*} \times \mathrm{P}_{\mathrm{pub}}+\alpha \times \mathrm{a} \times$ $\mathrm{h}_{2}{ }^{*} \times \mathrm{W} \times \mathrm{bP}+\beta^{*} \mathrm{R}^{*}$ and $\mathrm{V}^{\prime}=\alpha \times \mathrm{a} \times \mathrm{h}_{1}{ }^{*} \times \mathrm{P}_{\mathrm{pub}}+\alpha \times \mathrm{a} \times \mathrm{h}_{2}{ }{ }^{*} \times \mathrm{w} \times \mathrm{bP}+\beta^{*} \mathrm{R}^{*}$.C can compute $\mathrm{abP}=\left(\mathrm{V}^{*}-\mathrm{V}^{\prime}\right) /$ $\left(\alpha \times \mathrm{W} \times\left(\mathrm{h}_{2}{ }^{*}-\mathrm{h}_{2}{ }^{\prime}\right)\right)$.So $\mathrm{C}$ can successfully obtain the solution of the $\mathrm{CDH}$ problem.

\section{Comparative Analysis}

In this section, we have calculated and analyzed the security performance and efficiency of the proposed scheme. First, the proposed scheme can resist ESL attacks, however, the scheme[6-10] can not.Now, we describe the vulnerability of [6-10] for ESL attacks.

(1) In the efficient CLS phase of [6],the signature is $\sigma(\mathrm{U}, \mathrm{v}, \mathrm{w})$. The signature generate as follows:select random $r_{1}, r_{2} \in Z p^{*}$. Compute $R=g^{r 1}, R^{\prime}=g^{r 2}, v=H_{2}\left(M, R, R^{\prime}, P_{i}\right) \in Z_{p}^{*}, U=\left(x_{i}{ }^{*} v\right.$ $\left.+r_{1}\right) D_{i}, W=x_{i} V+r_{2}$. The adversary $A_{I}$ who knows $\left\{r_{1}, r_{2}, x_{i}\right\}$ can compute the partial secret key $D_{i}$ as $\mathrm{D}_{\mathrm{i}}=\mathrm{U} /\left(\mathrm{x}_{\mathrm{i}}{ }^{*} \mathrm{v}+\mathrm{r} 1\right)$. The adversary $\mathrm{A}_{\mathrm{II}}$ who knows $\left\{\mathrm{r}_{1}, \mathrm{r}_{2}, \mathrm{D}_{\mathrm{i}}\right\}$ can compute the secret key $\mathrm{x}_{\mathrm{i}}$ as $\mathrm{x}_{\mathrm{i}}=\left(\mathrm{w}-\mathrm{r}_{2}\right) / \mathrm{v}$.

(2) In the efficient CLS phase of [7],the signer generate the signature $\sigma(\mathrm{h}, \mathrm{V})$. The signature can generate as follows:compute $S I D=x * D I D$, Select random $r \in Z_{q}^{*}$ and set $R=g^{r}$. Compute $\mathrm{h}=\mathrm{H}_{2}(\mathrm{M}\|\mathrm{ID}\| \mathrm{R} \| \mathrm{PID}) \in \mathrm{Z}_{\mathrm{q}}{ }^{*}, \mathrm{~V}=\mathrm{r} * \mathrm{P}+\mathrm{h} *$ SID. The adversary $\mathrm{A}_{\mathrm{I}}$ who knows $\left\{\mathrm{r}, \mathrm{x}_{\mathrm{i}}\right\}$ can compute the secret key SID as $\mathrm{SID}=(\mathrm{V}-\mathrm{r} * \mathrm{P}) / \mathrm{h}$.

(3) In the CLS phase of [8], he signature is $\sigma(\mathrm{U}, \mathrm{V})$. The signature generates as follows: compute 
$\mathrm{Q}_{\mathrm{A}}=\mathrm{H}_{1}\left(\mathrm{ID}_{\mathrm{A}}\right) \in \mathrm{G}_{1}$. Select random $\mathrm{r} \in \mathrm{Z}_{\mathrm{q}}{ }^{*}$ and set $\mathrm{U}=\mathrm{rQ}_{\mathrm{A}} \in \mathrm{G}_{1}$.Set $\mathrm{h}=\mathrm{H}_{2}(\mathrm{~m} \| \mathrm{U}) \in \mathrm{Z}_{\mathrm{q}}{ }^{*}$ and Compute $\mathrm{V}=(\mathrm{r}+\mathrm{h}) \mathrm{S}_{\mathrm{A}}$. The adversary who knows $\mathrm{r}$ can compute the secret key $\mathrm{S}_{\mathrm{A}}$ as $\mathrm{S}_{\mathrm{A}}=\mathrm{v} /(\mathrm{r}+\mathrm{h})$.

(4) In the CLS phase of [9], the signer generate the signature $\sigma(\mathrm{U}, \mathrm{V})$. The signature generates as follows:select random $r \in Z_{q}{ }^{*}$. Compute $U=r P$, and $V=D_{A}+r_{2}\left(m, I_{A}, P_{A}, U\right)+x H_{3}\left(m, I_{A}\right.$, $\left.\mathrm{PK}_{\mathrm{A}}\right)$. The adversary $\mathrm{A}_{\mathrm{I}}$ who knows $\{\mathrm{r}, \mathrm{x}\}$ can compute the secret key $\mathrm{D}_{\mathrm{A}}$ as $\mathrm{D}_{\mathrm{A}}=\mathrm{V}-\mathrm{rH}_{2}\left(\mathrm{~m}_{\mathrm{ID}} \mathrm{ID}_{\mathrm{A}}\right.$, $\left.\mathrm{PK}_{\mathrm{A}}, \mathrm{U}\right)-\mathrm{xH}_{3}\left(\mathrm{~m}, \mathrm{ID}_{\mathrm{A}}, \mathrm{PK}_{\mathrm{A}}\right)$.

(5) In CLS phase of [10],the signature is $\sigma(\mathrm{V}, \mathrm{S}, \mathrm{R})$. The signature can generate as follows:Select random $\mathrm{r} \in \mathrm{Z}_{\mathrm{q}}^{*}$. Compute $\mathrm{S}=\mathrm{D}_{\mathrm{ID}} / \mathrm{S}_{\mathrm{ID}}, \mathrm{R}=\left(\mathrm{r}-\mathrm{S}_{\mathrm{ID}}\right) * \mathrm{P}$ and $\mathrm{V}=\mathrm{H}_{2}\left(\mathrm{M}, \mathrm{R}, \mathrm{P}_{\mathrm{ID}}\right)^{*} \mathrm{r}^{*} \mathrm{P}$. The adversary $\mathrm{A}_{\mathrm{I}}$ who knows $\left\{r, S_{I D}\right\}$ can compute the secret key $D_{I D}$ as $D_{I D}=S_{I D} * S$.

Then,we compare the efficiency of the proposed scheme with the schemes [6-10].For the performance comparison with respect to calculation cost,we considered the following notations. The performance comparison are showed in Table 1.

(i) $\mathrm{T}_{\mathrm{e}}$ : The time of executing bilinear pairing operation.

(ii) $\mathrm{T}_{\text {mul }}$ :The time of executing multiplication operation on elliptic curve.

(iii) $\mathrm{T}_{\mathrm{i}}$ : The time of executing modular inversion operation.

(iv) $\mathrm{T}_{\mathrm{s}}$ : The time of executing exponentiation operation.

(v) $\mathrm{T}_{\mathrm{h}}$ :The time of executing a map-to-point hash fuction.

(vi)G:The length of a point in G1.

(vii)Z:The length of a point in $\mathrm{Zq}^{*}$.

Table 1.The performance of the schemes.

\begin{tabular}{|l|l|l|l|l|}
\hline Scheme & Sign & Verify & ESL attack & Signature Length \\
\hline Scheme in [6] & $2 * \mathrm{~T}_{\mathrm{s}}+\mathrm{T}_{\mathrm{h}}+\mathrm{T}_{\mathrm{mul}}$ & $\mathrm{T}_{\mathrm{e}}+2 * \mathrm{~T}_{\mathrm{mul}}+2 * \mathrm{~T}_{\mathrm{i}}+\mathrm{T}_{\mathrm{s}}+\mathrm{T}_{\mathrm{h}}$ & Yes & $\mathrm{G}+2 * \mathrm{Z}$ \\
\hline Scheme in [7] & $\mathrm{T}_{\mathrm{s}}+\mathrm{T}_{\mathrm{h}}+2 * \mathrm{~T}_{\mathrm{mul}}$ & $2 * \mathrm{~T}_{\mathrm{e}}+\mathrm{T}_{\mathrm{mul}}+\mathrm{T}_{\mathrm{i}}+\mathrm{T}_{\mathrm{h}}$ & Yes & $2 * \mathrm{G}$ \\
\hline Scheme in [8] & $2 * \mathrm{~T}_{\mathrm{h}}+2 * \mathrm{~T}_{\mathrm{mul}}$ & $2 * \mathrm{~T}_{\mathrm{e}}+\mathrm{T}_{\mathrm{mul}}+2 * \mathrm{~T}_{\mathrm{h}}$ & Yes & $2 * \mathrm{G}$ \\
\hline Scheme in [9] & $2 * \mathrm{~T}_{\mathrm{h}}+3 * \mathrm{~T}_{\mathrm{mul}}$ & $4 * \mathrm{~T}_{\mathrm{e}}+3 * \mathrm{~T}_{\mathrm{h}}$ & Yes & $2 * \mathrm{G}$ \\
\hline Scheme in [10] & $3 * \mathrm{~T}_{\mathrm{mul}}$ & $2 * \mathrm{~T}_{\mathrm{e}}+2 * \mathrm{~T}_{\mathrm{mul}}+\mathrm{T}_{\mathrm{h}}$ & Yes & $3 * \mathrm{G}$ \\
\hline The proposed scheme & $4 * \mathrm{~T}_{\mathrm{h}}+5 * \mathrm{~T}_{\mathrm{mul}}$ & $3 * \mathrm{~T}_{\mathrm{e}}+2 * \mathrm{~T}_{\mathrm{mul}}+4 * \mathrm{~T}_{\mathrm{h}}$ & No & $2 * \mathrm{G}$ \\
\hline
\end{tabular}

The table shows that the bilinear operation of scheme [6] is the least, but the computational complexity is very high. Signature length and security performance which schemes [7-9] produces are similar. The scheme [10] makes small computational cost, but the signature length is long.Although our scheme produces some computational cost,however, it can resist ESL attacks and provable security in the random oracle model.

\section{Conclusion}

In this paper, we propose a new secure certificateless signature scheme. This new scheme can resist ESL attacks.Moreover, we prove the security of two types of adversaries in certificateless signature scheme.The proposed scheme provides unforgeability based on the hardness assumption of CDH problem.Therefore, this scheme can be used in many security applications.

\section{Acknowledgements}

This work was financially supported by the European Seventh Framework Program(FP7) 
(GA-2011-295222) and the National Sci-Tech Support Plan of China (2014BAH02F03).

\section{Reference}

[1] Shamir A. Identity-Based Cryptosystems and Signature Schemes[J]. Lecture Notes in Computer Science, 1995, 21(2):47-53.

[2] S. Al-Riyami and K. Paterson.Certificateless public key cryptography.Lecture Notes in Computer Science, vol. 2894, pages 452-473, Springer-Verlag, 2003.

[3] Yulei Zhang,Saifen Wang,Yongjie Zhang,et al. A new efficient certificateless signature scheme based on [J]. Computer Engineering and Applications.2010, 46(14):84-87.In Chinese.

[4] Huang X, Susilo W, Mu Y, et al.On the Security of Certificateless Signature Schemes from Asiacrypt 2003[M]//International Conference on Cryptology and Network Security.2005:13-25.

[5] Yum D H, Lee P J. Generic Construction of Certificateless Signature[C]//Information Security and Privacy:, Australasian Conference,ACISP 2004,Sydney,Australia,July 13-15, 2004.

[6] Zhang L, Zhang F, Zhang F. New Efficient Certificateless Signature Scheme[M]// Emerging Directions in Embedded and Ubiquitous Computing. 2007:692-703.

[7] Yufeng Li,Yupei Liu,Zhangfang Zhu.Efficient certificateless signature scheme [J].Computer Engineering and Applications.2011, 47(10):23-26.In Chinese.

[8] Yap W S, Heng S H, Goi B M. An Efficient Certificateless Signature Scheme[J]. Lecture Notes in Computer Science, 2006, 4097:322-331.

[9] Zhang Z, Wong D S, Xu J, et al. Certificateless public-key signature: security model and efficient construction[C]// Applied Cryptography and Network Security, International Conference, ACNS 2006, Singapore, June 6-9, 2006, Proceedings. DBLP, 2006:293-308.

[10] Xu Z,Liu X,Zhang G,et al.A Certificateless Signature Scheme for Mobile Wireless CyberPhysical Systems[J].International Journal of Computers Communications \& Control,2008, III(4):489-494.

[11] Tseng Y M, Tsai T T, Huang S S. Leakage-Free ID-Based Signature[J]. The Computer Journal, 2015, 58(4):750-757.

[12] Islam S H, Li F. Leakage-Free and Provably Secure Certificateless Signcryption Scheme Using Bilinear Pairings[J]. The Computer Journal, 2015, 58(10):2636.

[13] Islam S H. A Provably Secure ID-Based Mutual Authentication and Key Agreement Scheme for Mobile Multi-Server Environment Without ESL Attack[J]. Wireless Personal Communications, 2014, 79(3):1975-1991.

[14] Islam S H, Biswas G P. An improved ID-based client authentication with key agreement scheme on ECC for mobile client-server environments[J]. Theoretical \& Applied Informatics, 2012, 24(4):293-312.

[15] Lamacchia B, Lauter K, Mityagin A. Stronger Security of Authenticated Key Exchange[C]// International Conference on Provable Security. Springer-Verlag, 2006:1-16.

[16] Zhang L, Zhang F.A New Provably Secure Certificateless Signature Scheme[J]. 2008, 61(7):1685-1689.

[17] Pointcheval D, Stern J. Security Proofs for Signature Schemes[C]// International Conference on Theory and Application of Cryptographic Techniques. Springer-Verlag, 1996:387--398. 